\title{
Drug education in schools: laying the foundation for drug abuse prevention
}

Tay Bian How.

Asian Centre for Certification and Education of Addiction Professionals (ACCE), The Colombo Plan

Correspondence: email: bian.howtay@colomboplan.org

\section{Abstract}

Preventive drug education is the cornerstone in the combat against drug use, particularly in efforts to insulate the younger generations from succumbing to the lures of drugs. This paper elaborates the conceptual/theoretical framework for drug education in schools which includes the various effective approaches and essential components that are considered to be of paramount importance for effective preventive drug education in schools.

Keywords: Preventive drug education, drug abuse

\section{Rationale of preventive drug education in schools}

The preventive drug education programme of a country is the foremost strategy in combating its drug abuse problem. Therefore, utmost importance must be given to preventive drug education efforts in schools due to the following reasons. ${ }^{1}$

- Higher incidence of young drug abusers

- Schools have the advantage of providing a captive audience that covers almost everyone in the appropriate age range for primary prevention

- Schools are ideally located and equipped to provide many of the cognitive, affective and skill components that are considered essential for effective prevention

- Schools are the link between homes and the wider community, and provides a two-way medium of communication and influence

- Schools are micro-communities within which a wide variety of educational, environmental and policy strategies can be implemented with respect to drugs and other health issues

In view of the above mentioned facts, schools should pay attention to drug demand and supply reduction, although the focus is demand reduction. Schools certainly are able to influence the behavioural patterns of youth rejecting drug use. 


\section{Theoretical framework}

"Just as we shape and mould bonsai trees when they are young so that they develop strong, and increase in beauty and value as they mature .. so do we shape and mould our young in their formative years so that they develop strong moral strength and confidence as they mature". ${ }^{2}$

Prevention programmes need to begin early (in pre-school or elementary school), to be broad in scope, and to have a deep, pervasive impact on children. To be maximally effective, programmes need to begin early enough in the socialisation process to provide positive influences on development. This is because problem behaviours are generally manifested at early ages. Aggressive behaviour in pre-school, for example, is often a precursor of later difficulties. Thus, early interventions that seek to promote successful adjustment and adaptation to the academic and social environment of the school prior to the emergence of significant academic and social problems hold the greatest promise of being effective strategies for primary prevention.

Prevention programmes should be comprehensive, i.e. promote positive personal and social development along with acquisition of academic skills and intellectual growth. In other words, prevention programmes should attempt to create and maintain a positive social climate that facilitates socialisation, rather than attempt to compensate for a prevailing negative social climate. Furthermore, prevention programmes should be a natural and important part of the school curriculum.

For effective school-based drug education, its content and processes must focus on the major determinants of human behaviour. These include the enhancement of personal determinants of behaviour, namely cognitive, affective, skill, and behavioural components, and social determinants of behaviour, including social norms and behaviours in the school, family and community.

Ideally, prevention programmes should also be widespread. Drug education should be implemented routinely at all grade levels and repeated consistently to reinforce experiences as students progress through grades. Effective drug education is cumulative. Educational programmes should be implemented with adequate intensity of time and effort. This includes not just booster sessions, but also co-curricular programmes implemented after school. In developing countries, it is common for both parents to be employed, thus leaving the child without supervision during non-school hours. Without adult supervision, children are vulnerable and easily influenced by anti-social activities. The current school curricula are not well suited to the needs of most working families. Therefore, drug education in schools should include co-curricular activities. 
A review of the existing prevention models illustrates that they can be categorised into five different approaches or models as follows:

- Information model (cognitive)

- Affective education/interpersonal approach

- Social-learning model/behavioural approach

- Alternative model

- Comprehensive school approach

The information model assumes that children use drugs because they are ignorant of the dangers. Thus, the approach provides information about drugs to children and often employs 'scare tactics' to deliver its message. However, knowledge alone does not affect behaviour and 'scare tactics' alone do not work. Children are becoming more sophisticated and peer pressure is a strong counterbalance.

The affective education/interpersonal approach, or individual deficiency model, is based on the assumption that adolescents use drugs because they lack some essential trait or ability. Thus, the model emphasises values clarification, skills development, and improving self-esteem, self-worth, and self-concept. However, this model has shortcomings. Brief programmes reinforcing self-esteem and promoting skills development may not be sufficient to change lifetime experience, nor provide adequate information about drugs.

The social-learning model states that drug use usually starts in a group setting among peers or relatives. In other words, youth lack social competencies to resist. The social-learning model provides them with specific skills such as resilience development and refusal skills (how to say "no"). This model also establishes normative expectations for adolescents.

The alternative model is based on the theoretical assumption that providing adolescents with 'alternative highs' or skills and competency-building activities will help curb drug use. The alternative model is ineffective if it is the only intervention for adolescents. However, it is has proven to be effective for high-risk adolescents when used along with other approaches.

Earlier approaches relied on a primary, single-strategy method. The comprehensive school or 'thousand flowers' approach consists of many different prevention components co-ordinated and adapted to meet local needs. It reaches beyond the schools into family and community life and aims not only to provide information but also to promote sustained personal development. Most comprehensive programmes combine teaching strategies that reflect 
knowledge/information, affective, interpersonal, and social influences approaches. This coordinated, multicomponent approach has shown positive results and is currently used in most prevention programmes.

Besides giving students clear, concise information about the dangers of drug use, the comprehensive strategy promotes wellness among youth. Wellness is a person's quality of life including his or her physical, mental-emotional, familysocial, and spiritual health. Thus, a comprehensive drug-education programme not only provides information but also emphasises development of life skills, decision-making skills, resistance skills, and resiliency to help adolescents choose to be drug free.

"Life is no brief candle to me. It is a sort of splendid torch which I have got to hold for the moment and I want to make it burn as brightly as possible before handing it on to future generations". ${ }^{2}$

Life skills ${ }^{1}$ promote well-being, and they are learnt and practiced for a lifetime. These skills enable youths to become strong, have a sense of self, and resist external pressures. Thus, life-skills education promotes students' wellbeing, help them make responsible decisions, and resist harmful peer pressure. Students are taught to use assertive behaviours, such as saying "no" to drugs and influencing others to choose responsible behaviours.

Drug education must provide vulnerable young people with armour to protect themselves when exposed to vulnerable situations. Thus, a drug-education programme must include means to develop the protective factors that promote resilience. Resilience is the ability to recover from or adjust to change, pressure, and adversity. Resilient youth are stress resistant and invincible despite adversity. They are able to cope with misfortune without using drugs.

\section{Components for effective preventive drug education in schools}

Drug education in schools may be defined as the educational programmes, policies, procedures and other experiences that contribute to the achievement of broader health goals of preventive drug abuse and their adverse consequences impacting individuals and society. Preventive drug education should be considered in relation to both the formal and informal curricula in health, the creation of a safe and healthy school environment, the provision of appropriate health services and the involvement of the family and community in the planning and delivery of programmes.

\section{Education components}

- $\quad$ Equipping young people with the information, skills/attitudes, values and understandings about drugs 
- Clearly communicating policies and procedures that strive to achieve a safe and supportive school environment, including provision of care, counseling and support for all students and a cooperative approach among staff, students, parents, and related professionals/agencies and the police

- Strategies that ensure all members of the school community contribute to and support school policies and procedures for dealing with drug related issues

- Appropriate professional development/training for relevant staff in preventive drug education

- Information and support for parents, particularly parents of students involved in drug use

- Mechanisms for ongoing monitoring and review of the school's approach to drug education and incident management

\section{Intervention components}

- Policy and procedures for drug incidents that consider the context of the student's life, family situation, mental and emotional health, intellectual ability and the degree to which they may be in control of their actions and decisions

- Immediate and longer term options for responding to drug incidents that protect the health of students and the school community

- A plan for managing drug incidents consistent with laws, regulations and policies

- A communication strategy for drug incidents that ensures all staff are aware of school and/or system-wide procedures for contacting and responding to the media

- A directory of professionals and agencies, including the police, who can provide professional development, advice and resources

- Protocols with professionals and agencies, including the police, to formalise and strengthen cooperative liaison and referral arrangements

- Support for students involved in drug incidents that maintain their engagement in education programmes

- Maintenance of records of drug incidents with due attention to the protection of the rights and privacy of all those who may be involved, and their usefulness in monitoring the effectiveness of policies 
The essential components in a comprehensive drug education programme are implemented through the curriculum, co-curriculum, teacher training, early intervention activities and involvement of the parents and community in preventive drug education. Past preventive drug education programmes had focused primarily on the imparting of factual knowledge on the variety of drugs without actually dealing with the situations that lure vulnerable adolescents to experiment. It was found that factual knowledge alone was insufficient to change adolescents with life skills to deal with situations that could lead them towards deviant behaviour. Adolescents are provided with ways to enhance selfesteem, communication skills, assertive skills, decision-making skills, stress management skills and refusal skills along with knowledge and role playing experiences related to drugs and staying away from them.

\section{Curriculum}

The long-term strategy used to implement preventive drug education in schools is to integrate drug abuse preventive education throughout the school curriculum. As in many developing countries, the schools follow a uniformed curriculum that is centrally developed by the Ministry of Education and it has been found that preventive drug education is most appropriately implemented in the health education/science curriculum.

\section{Co-curriculum}

Preventive drug education is also integrated into co-curricular activities, which complement and supplement the academic curriculum. Another objective of the co-curriculum is to encourage school children to get involved in activities which promote a healthy lifestyle as an alternative to drug abuse. The co-curricular programme is effective for the latchkey students as there is a considerable increase in the number of dual career and single-parent families and a decrease in the traditional, extended family unit for childcare in Southeast Asia, thus leaving them to care for themselves. Hence co-curricular activities will ensure that they participate in healthy alternatives in schools under the supervision of teachers. Co-curricular activities are essential in nurturing and instilling 'esprit de corps' among the students. They are also intended to teach students to be responsible, disciplined, independent and skilled in areas of their choice.

\section{Teacher training and teaching methodologies}

Since drug education will be integrated into the curriculum, it is most appropriate to train at teacher training colleges. Moreover, teachers qualified in drug education are needed for effective implementation of preventive drug education programmes. Hence, a module of drug education is incorporated into the teachertraining curriculum so as to provide all teachers with the basic knowledge of preventive drug education and essential skills to handle students at risk. Besides 
the pre-service training of teachers, in-service training courses could be conducted regularly to orientate and update teachers on drug abuse. ${ }^{3}$

In teacher training courses related to drug education, special emphasis has been placed on teaching/instructional methods. The traditional method has been the didactic approach, which is heavily concentrated in merely imparting knowledge via the lecture/discussion method. This approach assumed that once students have adequate and accurate knowledge about drugs and their effects, they would behave rationally and choose not to use drugs. On the contrary, it has been well documented that increased knowledge does not necessary lead to behavioural changes among adolescents. Moreover, the information-based approach leads to students' rejection of messages and sources. ${ }^{3}$

In the early years of the drug crisis particularly in the 1960s and '70s, scare tactics were often used to scare students by presenting the statistics, risks and consequences of drug use in an overly dramatic fashion, thereby undermining the credibility of the information presented. Hence, it was not only ineffective but in some ways also counterproductive.

Since drug education aims to instill behavioural change, the most effective teaching method in imparting knowledge and skills to the students is the life skills approach that emphasises on experiential learning. A The life skills has the potential to lead to better health and drug education learning outcomes and may ultimately influence student behaviour against drug abuse. Life skills are best taught through interactive methods and are most effective when applied and practiced in potential drug use situations that are relevant and meaningful to the social situations of students. It is more effective if delivered by teachers in classrooms in a non-threatening and learner-friendly environment.Thus, drug education is more successful when it is student focused and adopts interactive methods with experiential learning and small group work as its basis. The life skills approach to drug education will provide drug information with the aim of developing attitudes, values and skills in students. These include skills for increasing self-esteem, setting realistic goals, coping with anxiety, resisting pressures, communicating effectively, making decisions, managing conflict, and dealing assertively with social situations in which drugs are offered.

\section{Involvement of the community}

"If a young boy is aware, he himself is only aware; if a young lady is aware, a family is aware; if a teacher is aware, the whole community is aware".'

The role of parents as primary educators should be recognised and supported by schools by working in partnership with parents. Partnership with parents and community help to integrate consistent and relevant preventive drug education 
messages into the home and the community and enable a greater awareness of issues related to drug abuse by students and their families.

Programmes that are implemented and initiated in consultation with parents are not only more successful, but also empower them. Parents often have difficulty discussing drug related issues with their children, yet parents can be the most trusted and preferred source of information for young people.

Schools can assist parents by providing information related to drug abuse issues as a part of the school approach. Schools working in partnership with parents remove some of the anxiety parents experience from the expectation that drug education is their sole responsibility. Further, such programmes have the potential to provide parents with skills and knowledge to initiate and carry out informed discussions with their children.

Parents are also important because families are a primary socialisation source, and because parental opinion can either, reinforce or go against the messages of drug education programmes. Parents are important also as their opinions contribute to community norms about drug use and community support for preventive drug education.

\section{Managing drug related incidents}

Statistics show that the popular reasons influencing adolescents initiating drug use are peer pressure, curiosity, thrill seeking and a lack of interpersonal skills. Evidence has shown that adolescents are at a much higher risk than others. The youth are more vulnerable and face higher probabilities of a problem occurring. Hence early intervention programmes or efforts to manage drug related incidents are essential to identify those at risk and already experimenting drug use so as to enable students' assistance programmes to be implemented by the school's counsellors.

Random urine screening in schools has been implemented in some Asian countries as a preventive measure and a deterrent to misuse of drugs. It is merely an exercise in early intervention. Those students who are identified to be at risk are required to undergo counseling sessions by the school counsellor. With the early intervention programme, it is hoped that potential drug abusers amongst students would be quickly identified and the problem nipped in the bud.

\section{Evaluation}

Evaluating the effectiveness of a comprehensive drug-prevention programme is important. Inevitably, concerned parties will want to know if the goal of the programme, i.e. to provide the students with knowledge and skills that will help them remain drug free, is achieved or not. Often in developing countries, evaluation is the most neglected tool of drug-prevention practitioners. Some 
believe that they cannot afford to spend time analysing past efforts when they are overburdened with new activities. Others are convinced that evaluation is a highly technical thereby requiring the services of experts at a cost that many organisations cannot afford. Simple evaluation techniques, however, require little time, cost and effort.

There are three major types of evaluations: process, outcome, and impact. Process evaluations describe and assess programme materials and activities. Examination of materials is likely to occur while programmes are being developed, as a check on the appropriateness of the programme's approach and procedures. Examining the implementation of the activities of programmes is also an important form of process evaluation. Outcome evaluations, on the other hand, assess programme achievements and effects. Outcome evaluations study the immediate or direct effects of the programme on participants. The scope of an outcome evaluation can extend beyond knowledge and/or attitudes. Finally, impact evaluations examine beyond the immediate results of policies, instructions, or services to identify long-term as well as unintended programme effects.

These three methods correspond to three broad purposes of programme evaluations, as follows:

i. To describe how the curriculum and related activities are delivered (process evaluation)

ii. To assess whether students have understood information about drugs and their consequences (outcome evaluation)

iii. To assess whether students' attitudes and/or behaviour have changed over time (impact evaluation) With these three purposes in mind, the following indicators can be used to evaluate the comprehensive drugeducation programme ${ }^{3}$.

- The extent to which students exhibit positive changes in attitude, knowledge, and behaviour

- The extent to which students participate in alternative and co-curricular activities

- The extent to which students exhibit positive changes in education/schooling behaviours

- Positive changes in school and drug-related statistics

Often, it takes time for a programme and its components to stabilise and begin to show results. Evaluation in this field can be difficult, but it is indispensable. Capitalising on successes and avoiding past mistakes make evaluation important to most organisations. 


\section{Conclusion}

To meet the challenges posed by the problem of drug use in a context where it is fast becoming more complex and multifaceted, it is of paramount importance that countries implement a comprehensive preventive drug-education programme as the foundation to combat the drug scourge in the region. In essence, there must be a commitment by the government of the country towards the transformation of its drug prevention programme to incorporate several components by the means of a preventive drug-education policy in schools. The policy and programme in schools should be in accordance with the national policy against drug use. It is hoped that this paper will provide the justification needed for countries to examine their existing preventive drug education programmes and make amendments to their programmes in achieving better outcomes.

\section{References}

1. Tay, Bian How 'Skills for Drug Prevention'; In: Report of the IV Global Conference on Drug Prevention; The Colombo Plan Drug Advisory Programme 2002

2. The Colombo Plan Drug Advisory Programme; 'Best Practices on Drug Abuse Prevention in Asia'; Colombo, Sri Lanka; June 2001.

3. Tay, Bian How 'Drug Prevention Education in Schools': the Malaysian Experience, Drugs: education, prevention and policy 1999;6(3):34351.

Doi: http://dx.doi.org/10.1080/09687639997016

4. Tay, Bian How; 'Strategies and Best Practices in Drug Abuse Prevention in the Asia and the Pacific'; Ceylon Daily News; 26 June 2003.

5. UNODC; 'School-based Education for Drug Abuse Prevention'; N.Y. 2004 